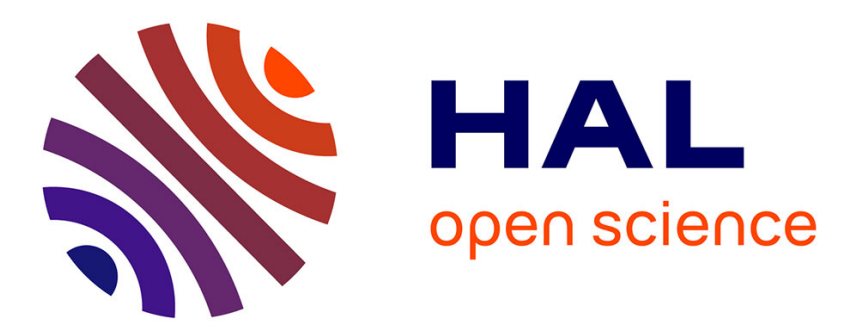

\title{
Comparing wavelet transforms for recognizing cardiac patterns
}

Lotfi Senhadji, Jean-Jacques Bellanger, Guy Carrault, Gianfranco Passariello

\section{To cite this version:}

Lotfi Senhadji, Jean-Jacques Bellanger, Guy Carrault, Gianfranco Passariello. Comparing wavelet transforms for recognizing cardiac patterns. IEEE Engineering in Medicine and Biology Magazine, 1995, 14 (2), pp.167-173. 10.1109/51.376755 . inserm-00152896

\section{HAL Id: inserm-00152896 https://www.hal.inserm.fr/inserm-00152896}

Submitted on 26 Jun 2007

HAL is a multi-disciplinary open access archive for the deposit and dissemination of scientific research documents, whether they are published or not. The documents may come from teaching and research institutions in France or abroad, or from public or private research centers.
L'archive ouverte pluridisciplinaire HAL, est destinée au dépôt et à la diffusion de documents scientifiques de niveau recherche, publiés ou non, émanant des établissements d'enseignement et de recherche français ou étrangers, des laboratoires publics ou privés. 


\title{
Comparing Wavelet Transforms for Recognizing Cardiac Patterns
}

\author{
L. Senhadji, G. Carrault, J. J. Bellanger, and G. Passariello* \\ Laboratoire Traitement du Signal et de I'lmage, INSERM, Université de Rennes I \\ * Grupo de Bioingeneria y Biofisica Aplicada Universidad Simon Bolivar
}

Published in This paper appears in: IEEE Engineering in Medicine and Biology Magazine, Publication Date: Mar/Apr 1995, Volume: 14, Issue: 2, On page(s): 167-173.

ISSN: 0739-5175

References Cited: 15

CODEN: IEMBDE

INSPEC Accession Number: 4930363

Digital Object Identifier: 10.1109/51.376755

This material is presented to ensure timely dissemination of scholarly and technical work. Copyright and all rights therein are retained by authors or by other copyright holders. All persons copying this information are expected to adhere to the terms and constraints invoked by each author's copyright. In most cases, these works may not be reposted without the explicit permission of the copyright holder. 


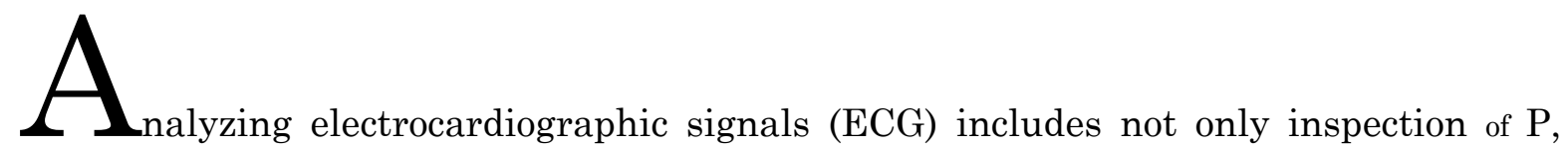

QRS and T waves, but also the causal relations they have and the temporal sequences they build within long observation periods. In fact, the spatio-temporal patterns must be described through shape features as well as complete time occurrence distributions in order to match the observed data with the underlying processes. It has been demonstrated recently that in depth knowledge can be qualitatively modeled by means of anatomofunctional decompositions [1] and can be used to simulate complex phenomena (i.e., reentry, block, etc.) and to provide a real understanding of their behavior at different abstraction levels. However, the on-line examination of ECG recordings can only be carried out if the relations between data events and the model are available. In other words, signal processing techniques must feed the model with accurately estimated features to discard the non relevant interpretations. This task is difficult to achieve because of the composite nature of the ECG (i.e., a combination of signal and noise) and its non stationary behavior. These characteristics motivated the approach we de-scribe, which makes use of wavelet transforms (WT) or time scale representations. These new tools have already been applied in ECG analysis for enhancing late potentials [2-3], reducing noise [4], and QRS detection [5]. In this article, we limit our study to recognizing normal and abnormal beats, and assume that a prior segmentation has been performed. It differs from previous work by addressing four main questions: 1) what is the most appropriate WT to use? 2) what are the most relevant features for efficient encoding of cardiac patterns? 3) what decomposition levels must be retained? 4) does WT improve the recognition process?

The first issue is critical in all application areas. There is no theoretical answer at the moment, and the only technique at our disposal is to compare the results provided by several wavelet families. Questions 2 and 3 have been examined by considering two stages:

(a) a characterization phase based on a principal component analysis (PCA) [6], which allows us to jointly represent and interpret the objects (i.e., the cardiac complexes) and the descriptive variables; and (b) a discrimination step by means of a linear discriminant analysis (LDA) [7]. This last stage leads us to identify the variables capable of separating the patterns, the objective being to derive the most discriminant decomposition levels. A supervised procedure is first applied on a learning set. The resulting performance is further tested on an additional set of patterns. The fourth issue has been considered by comparing the best solution provided by the WT with classical signal descriptions.

This article presents the wavelet transforms that we have used and some of their basic properties. The ECG beat recognition is also addressed. The analysis is carried out by combining several parameterizations (distributions of energy and extrema along the decomposition levels) according to the wavelets under study. This section includes three parts: (a) comparison among descriptors issued from orthonormal wavelets; (b) comparison with two other wavelet families and (3) comparison with classical parameter sets.

\section{Wavelet Transforms}

The wavelet transform of a signal, $\mathrm{S}(\mathrm{t})$, corresponds to its decomposition with respect to a family, F, of functions obtained by dilations (or contractions) and translations of an analyzing wavelet denoted $Y$ [8]. The coefficients Da,b deduced from this decomposition are expressed by:

$$
D_{a, b}=\int_{-\infty}^{+\infty} S(t) \Psi_{a, b}^{*}(t) d t(1)
$$

where the superscript * denotes the complex conjugate. The set of Da,m with $a$ non zero and $\boldsymbol{b}$ real, defines the continuous wavelet transform. 
There are several wavelet families, each corresponding to a different representation of the analyzed signal.

The decomposition based on orthonormal wavelets leads to a non redundant description. With $\tilde{\Psi}_{a}(t)=\frac{1}{\sqrt{|a|}} \Psi^{*}\left(\frac{-t}{a}\right), D_{a, b}$ can be written: $D_{a, b}=\int_{-\infty}^{+\infty} S(t) \tilde{\Psi}_{a}(b-t) d t$

where $\mathrm{S}(\mathrm{t})$ is the input. $D_{a, b}$ is thus the output, at time b, of the filter whose impulse response is $\tilde{\Psi}_{a}(t)$, and where $a$ allows sets the bandwidth. This transformation acts on the signal as a filter bank whose frequency characteristics are linked to $\Psi(\mathrm{t})$ and to the parameter $a$. In other words, using a prior knowledge of the signal under study, we can focus on a subset of scale parameters in the analysis. This interpretation becomes obvious when the WT is associated to a multiresolution analysis, where the decomposition, with respect to an orthonormal base of $\mathrm{L}^{2}(\mathbb{R})$, is replaced by an iterative scheme based on high pass and low pass filtering followed by a downsampling [9]. S(t) is then decomposed into a discrete set of orthogonal details (the outputs of the high pass filters) from which the exact reconstruction of the signal can be carried out. Nevertheless, up to now, neither knowledge of the processes generating the signals nor the specification of objectives at hand allow to us to a priori assess the best wavelet to be used. Experiments must be conducted on simulated or real data and compared by means of objective criteria. Three comparative studies have been carried out to gain more insight into this problem. They use:

(1) the compactly supported wavelet bases of $L^{2}(\mathbb{R})$ introduced by Daubechies [10] and associated with minimal phase filters

(2) a non orthogonal base of $L^{2}(\mathbb{R})$ derived from a cubic spline wavelet [11] with exponential decay; the corresponding low pass filter is symmetric and the high pass filter is antisymmetric (3) a "Morlet type" wavelet $\Psi(\mathrm{t})$, a complex valued function expressed by:

$$
\Psi(t)=C\left(1+\cos \left(2 \pi f_{0} t\right)\right) \exp \left(2 i k \pi f_{0} t\right)
$$

where, $|t| \leq 1 / 2 f_{0}, \mathrm{k}$ is an integer different from $-1,0,1 ; f_{0}$ is the normalized frequency, and $\mathrm{C}$ is the positive constant such that the energy of $\Psi$ is equal to 1.

The two first wavelets make use of the dyadic sequence $(2-\mathrm{j}, \mathrm{j}>0)$ as scale parameters, and the equivalent filter bank is then completely determined. For the third wavelet, the scales have been chosen heuristically to fully describe the relevant signal components: $\mathrm{k}=2, f_{0}=$

0.01 , and $a_{i}=\frac{1}{1+i \Delta / f_{0}}, 0 \leq i \leq 50, \Delta=0.002$.

The basic properties of the wavelets applied here have been explored in [12], with emphasis on the local regularities of the analyzed signals. It has been shown, for the spline wavelet (2), that the decomposition maxima encapsulate the most important part of the signal information. The properties of the complex wavelet (Eq. 3) have been studied in [13]. It has been demonstrated that the inflexion points and the maxima of $\mathrm{S}(\mathrm{t})$ are, respectively, the maxima and minima (with respect of the time localization variable b) of the modulus square of its decomposition. In addition, a local extremum of $\mathrm{S}(\mathrm{t})$ corresponds, in the transformed domain, to a zero crossing (with modulo $\pi$ ) of the phase.

\section{Recognizing Isolated Beats}

Our study was conducted on a set of 53 patterns sampled at $300 \mathrm{~Hz}$ and previously segmented by temporal windowing (these signals are part of the European ECG data base and 
were provided by the Istituto di Fisiologia Clinica, Pisa). The signals were normalized in energy to take into account the disparity in magnitude due to the different amplifications used during acquisition as well as the variations induced by the lead sites (the signals come mainly from DI and V6 leads). The isolated signals include 20 normal beats, 13 premature ventricular contractions (PVC) and 20 patterns with an S-T segment deviation (these last ones were labeled as ischemic). Each beat is described by about 300 samples.

The ECG decomposition depicts different energy partitions for resolution levels of the beats under study. The abnormal waveforms (PVC and ischemic beats) are localized on the coarser levels when the normal patterns appear on the finest scales [13]. This result is illustrated in Fig. 1, where the orthonormal wavelet has been applied to a sequence of normal cardiac cycles containing one premature ventricular contraction. Figure 2 provides a more detailed view of the decomposition for an isolated normal beat. Figure 3 shows an- other common representation in time scale (for simplicity, the index $\mathrm{i}$ is used instead of ai). The major constituents, e.g., QRS waves, are enhanced due to their fast transitions when the other components are less visible These examples point out that, from decomposition at another level, no additional information is obtained. Consequently, based on our experience, the number of decomposition levels was set at 9, 6, and 10 for the wavelets (1), (2), and (3), respectively.

Comparing parameters derived from orthogonal wavelets

The feature sets include the energies estimated at each resolution level and the local extrema (value and location) of the decomposition.

Description by energy

With reference to [10], the wavelet corresponds to $\mathrm{N}=6$ (which means that the length of the filter is $2 \mathrm{~N}=12$ ). The feature vector components are the energy of each detail level (denoted ENi). The PCA applied to the full set of signals suggests retaining the two first principal axes (a significant jump is observed between the second and the third eigenvalues). The inertia extracted by these two factors is approximately 68 percent, and the quality of the representation of the objects (i.e., the beats) and the variables $\mathrm{ENi}$ is 0.94 , and 0.7, respectively. However, discriminant analysis shows that at least 3 variables are required to obtain a good separation (96 percent) among the 3 groups (one false classified pattern exhibits a low elevation of the S-T segment Table I). The most discriminant variables are the energies related to levels 8,7 , and 3 . This analysis confirms the visual inspection of the decomposition previously mentioned. The abnormal patterns have their main projections on levels 7 and 8; the most important contributions appear on level 3 for the normal beats.

Description by extrema

The following operations were performed: (a) signal decomposition on 9 levels; (b) selection of the global extremum at each level ; and (c) signal reconstruction from the extrema. Figure 4 illustrates these operations on 3 patterns (representing each group) for 3 functions $\Psi$ corresponding to $\mathrm{N}=3,6$ and 10 . The reconstruction based on the extrema (i.e., only one point within each decomposition level is kept) accentuates the peaks for $\mathrm{N}=3$ and depicts an oscillating behavior for $\mathrm{N}=6$ and 10. These phenomena, unrelated to the ob- served signals, are only due to the wavelet regularity characteristics. To show this dependence more clearly, the similarity between the original and reconstructed patterns was examined. Table II points out that their correlation coefficient ranges from 0.74 to 0.96 , the best results obtained with $\mathrm{N}$ $=3$ and $\mathrm{N}=6$. Correlations between the three reference waveforms and all the reconstructed ones has also been calculated (the maximal values are re- ported in Table III). For the pairs (normal, PVC) and (normal, ischemic), the lowest correlation coefficients are provided by $\mathrm{N}=$ 
10 and $\mathrm{N}=6$. Conversely, for the pair (PVC, ischemic), $\mathrm{N}=3$ gives the lowest correlation coefficient. However, these results emphasize that pattern recognition can be achieved from the extrema representation. The wavelet corresponding to $\mathrm{N}=6$ has been retained based on computation time considerations. Only the global extremum of each detail level is necessary, because the relative time locations of the wave components do not bring additional discrimination insights for the data set under consideration. This global absolute value represents in some way the correlation between the raw signals (original beats) and the base vectors of the detail subspaces and provides more information than its position.

The same statistical analysis has been carried out on the whole set of beats. The PCA leads to results equivalent to those reported above with a factorial space of dimension 3 . The LDA provides a discrimination rate of 98 percent when in- cluding the maximum values of levels 8,3 , and 7 , but the three groups of patterns are completely separated when information of level 2 is added.

Discussion

These results show that high performance is obtained by the two descriptions using a very reduced number of features. These descriptors are coherent in terms of resolution levels used to better discriminate the three groups of waveforms. The description by the maximum absolute value at each level performs slightly better than the energy descriptors. The latter take into account only the power distribution of the analyzed frequency bands, and not the shapes of the patterns. Two signals with the same energy distributions in the spectral bands resulting from the decomposition will be represented by the same feature vector. The maximum value description provides more insight into the shapes. The deviations from normal beats are here characterized by an energy localized in low frequency, but the pattern morphologies are also different. However, the maximal absolute values are insensitive to phase changes and remain a rough description of the signal.

Two additional LDA have been con- ducted to see if other improvements can be obtained. The first was applied by considering the sign of the global extremum value at each detail level. This analysis showed that strong variations are introduced within each group of patterns, and the corresponding clusters were no more coherent when represented in the factorial space. The sign acts as a perturbation on the whole set of objects. The second analysis was based on introduction of the three most important local extrema values (unsigned) at each level. The discrimination performance decreased whatever the levels taken into account. However, this performance decrement does not mean that this information (as well as the time locations) cannot play a role in other applications (or in other diseases), but that only here the maximal absolute values (computed at levels 3 to 8 according to the results) are sufficient for discrimination purposes.

Comparing with other wavelet families

The same approach (i.e., representation by a global maximum) has been applied to the decompositions provided by the spline wavelet (6levels) and the complex wavelet (10 levels). In both cases, the frequency band of the signal was pre- served. The classification rate is better for the first, a result expected because the complex wavelet leads to highly correlated (or redundant) maxima representation. When compared to the previous orthonormal base, the discrimination performance remains very poor. As mentioned above, these wavelets give additional cues as to the time localization of the largest slopes in the signals. This information was integrated into the feature set, with the time reference being the position of the maximum on the first level. The relative time position of the maximum (the difference between the maximum position and the time reference) at each level was then computed. Every pattern was described by an 11-dimensional vector for the spline wavelet (6 maxima and 5 relative time positions) and a 19-dimensional feature set for the complex wavelet (10 
maxima and 9 relative time positions). On this basis, the spline wavelet leads to results equivalent to the Daubechies, while the performance of the complex wavelet still remains lower.

Comparing with classical descriptors

This comparison was made by allocation of complementary patterns into the discriminant subspace resulting from a learning phase using the same data set. The time scale descriptions were based on the following features: 9 maxima (issued from the compactly supported wavelet); 6 maxima and 5 relative time positions (spline wavelet); 10 maxima and 9 relative time positions (complex wavelet).

The variables extracted from the temporal analysis of the patterns were: the maximum magnitude of the $\mathrm{P}, \mathrm{QRS}$ and $\mathrm{T}$ waves, and the $\mathrm{PQ}$ and $\mathrm{ST}$ interval durations. The representation, based on the power spectral density, was obtained from: (1) the percentage of energy present in the theoretical P, QRS, and T frequency bands [141 with respect to the total pattern energy, and (2) the mean and median frequencies. The quality of the discrimination provided by different feature sets was evaluated by constituting two sets: the training data ( 2.5 beats) and the test set (28 cardiac complexes). The only requirement for the definition of the first set was the quasi uniform representation of each group (normal, PVC, ischemic patterns). The random trials were repeated 20 times and the mean rate of well-classified patterns computed. This procedure was necessary because of the reduced size of the population under study, which excluded any estimation of the classification error. The rate of mean, maximum, and minimum correct classification resulting from these trials are reported Table IV. These results emphasize that the best performances are achieved when using the two first wavelets (i.e., (1) and (2)) and that the temporal and spectral descriptions lead to results quite similar to those obtained by means of the complex wavelet.

\section{Conclusions}

Our study made use of wavelet trans- forms to describe and recognize isolated cardiac beats. The choice of the wavelet family as well as the selection of the analyzing function into these families have been discussed. The criterion used in the first case was the correct classification rate, and in the second case, the correlation coefficient between the original pattern and the reconstructed one. Two types of description have been considered -the energybased representation and the extrema distribution estimated at each de- composition leveland their quality has been assessed by using principal component analysis. Their capability of discrimination between normal, PVC, and ischemic beats has been studied by means of linear discriminant analysis. This work leads also, for the problem at hand, to the identification of the most relevant resolution levels. The results (to be confirmed on larger data sets) can be summarized as follows:

(1) the detail levels that contribute to the quality of representation (PCA) and discrimination (LDA) are the same.

(2) the compact support wavelet and the spline wavelet lead to efficient recognition rules based on the extrema detected at each decomposition level. They show a better behavior than classical features computed in the temporal or spectral do- mains.

(3) the complex wavelet has a lower performance. However, it does not introduce the dyadic constraint and, as such, can be more relevant in other situations such as the detection of epileptic transients [15].

\section{Acknowledgment}

The authors are grateful to J.L. Coa-trieux for his suggestions and comments on this paper and to all the partners of the KISS European project (Knowledge Inter- active Signal Monitoring System, AIM Program). 


\section{References}

1. Siregar P, Coatrieux JL, Mabo P: How can deep knowledge be used in CCU monitoring? IEEE Eng. Med Biol 12(4): 92-99, 1993.

2. Tuteur F: Wavelet Transform in signal detection. Proc Int. Conf. IEEE ASSSP, New York, 1435- 1438, 1989.

3. Meste 0, Rix H, Jane R, Caminal P: Détection battement par battement de potentiels tardifs dans les Electrocardiogrammes a haute amplification. Innovation et Technologie en Biologie et Médecine 12(5): 517-531, 1991.

4. Senhadji L, Bellanger JJ, Carrault G, Coa-trieux JL: Wavelet analysis of ECG signal. Proc Int. Conf. IEEE EMBS, Philadelphia, 811-812, 1990.

5. Kadambe S, Murray R, Boudreaux-Bartels GF: The dyadic wavelet transform based QRS detector. Proc Int Conf. ASILOMAR, 1992.

6. Celeux G, Diday E, Govaert G, Lechevallier Y, Ralambondrainy H: Classification automatique des données, environnement statistique et informatique. Dunod Informatique, Bordas Ed., Paris 1989.

7. Romeder JM: MCthodes et programmes d'analyse discriminante. Dunod Ed., 1973.

8. Martinet RK, Morlet J, Grossmann A: Analysis of sound patterns through wavelet transform. J Pat Recog and Artif Intel. 1: 273-301, 1987.

9. Mallat SG: A theory of multiresolution signal decomposition: the wavelet representation. IEEE Trans PAMI 2(7): 674-693, 1989.

10. Daubechies I: Orthonormal basis of compactly supported wavelets. Comm Pure Applied Math. 41: 909-996, 1988.

11. Mailat SG, Zhong S: Complete signal representation with multiscale edges. Robotics Research Technical Report no. 483, New York University Courant Institute of Mathematical Sciences, December 1989.

12. Mallat SG, Zhong S: Characterization of signals from multiscale edges. IEEE Trans PAMI 14: 710-732, 1992.

13. Senhadji L: Approches multiresolution pour I'analyse des signaux non stationnaires. Ph.D. Thesis, Université de Rennes I, France, 1993.

14. Tbakor N,Webster J, Tompkins W: Estimation of QRS complex power spectra for design of a QRS filter. lEEE Trans BME 31: 770-778, 1984.

15. Senhadji L, Dillenseger JL, Rocha C, Wendlling F, KiniC A: Wavelet analysis of EEG for mapping of epileptic event. To be published in the Special Issue "Wavelet Transforms in Biomedical Engineering.” Ann Biomed Eng. 23(5):543-552, 1995. 


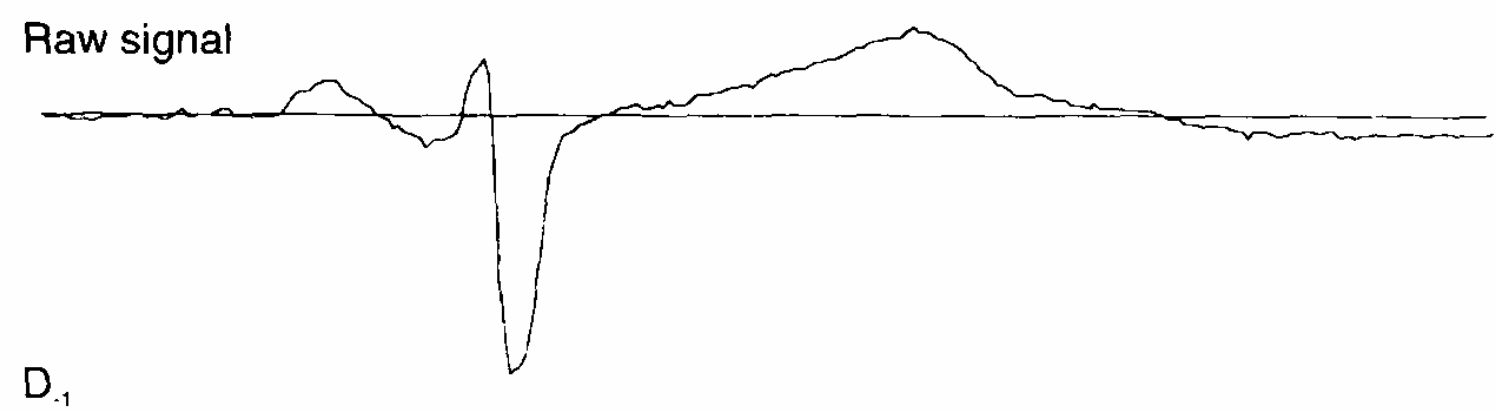

$D_{-2}$
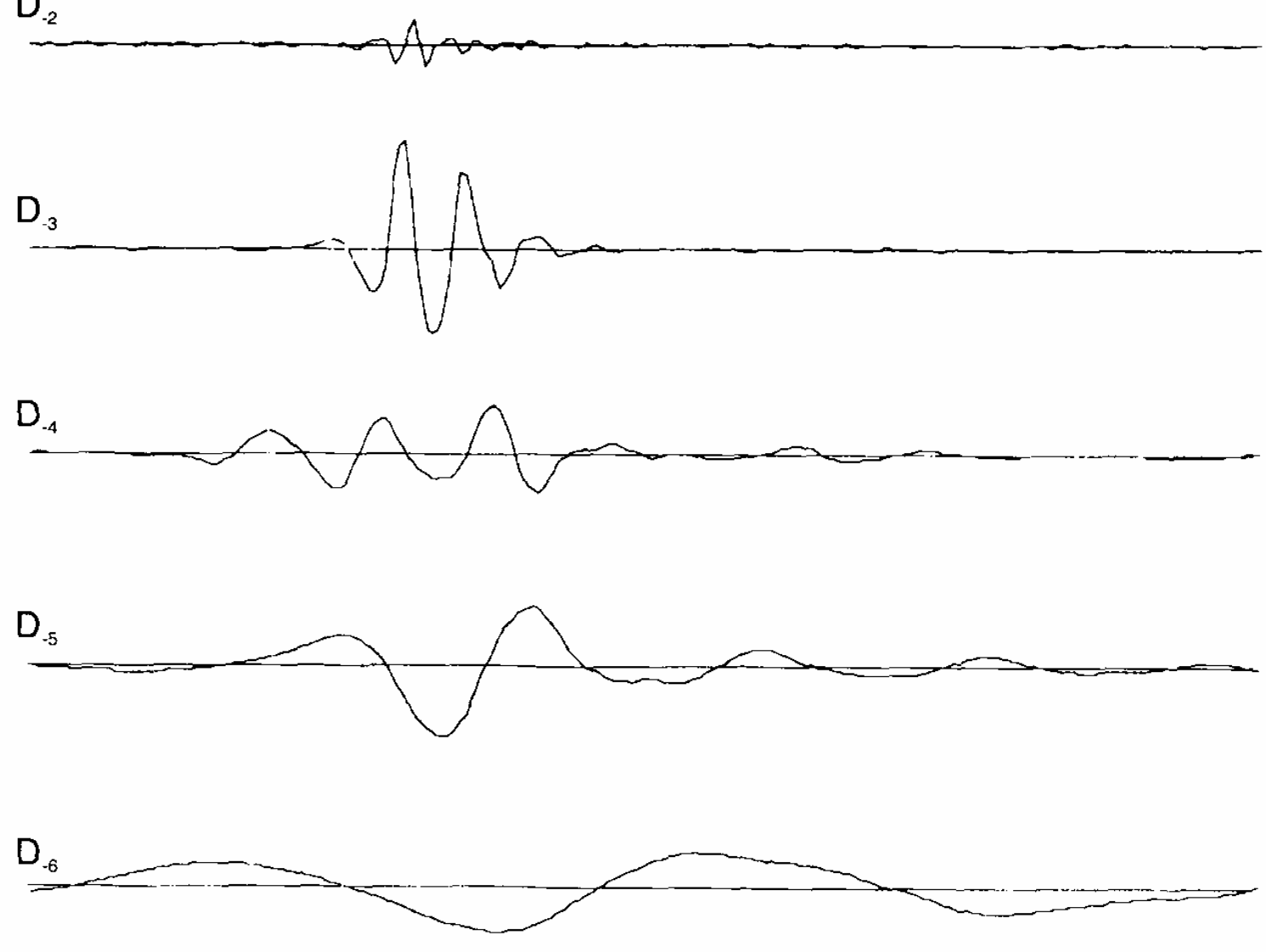

$\mathrm{D}_{-7}$

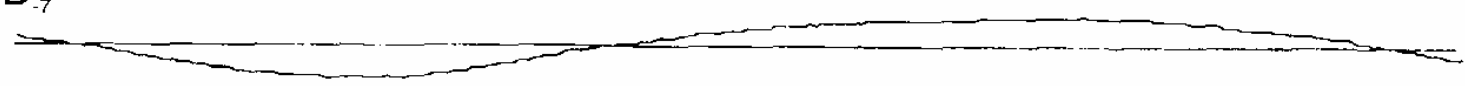

$\mathrm{D}_{-8}$

$D_{\cdot}$

1. Orthonormal decomposition of an ECG segment composed of normal beats and a PVC. Top (the raw signal). The detail levels are depicted below (from D-1 to D-9, where D-j is the detail at the resolution $2^{j}$ ). It can be seen that the PVC appears clearly on D-6 and D-7. 


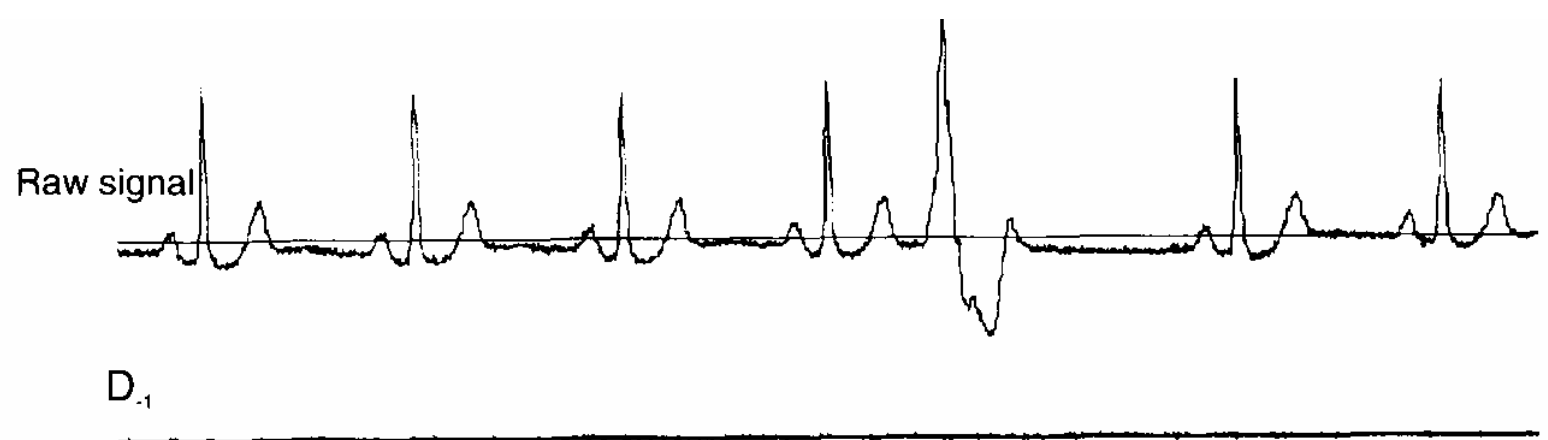

$\mathrm{D}_{-2}$
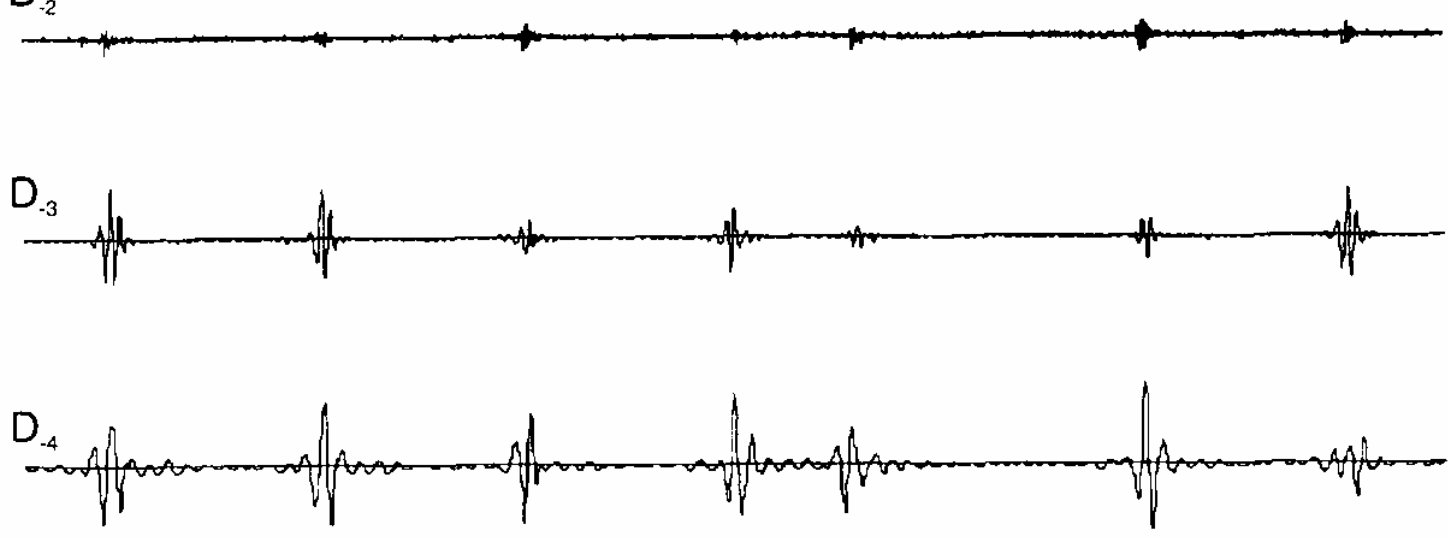

D.
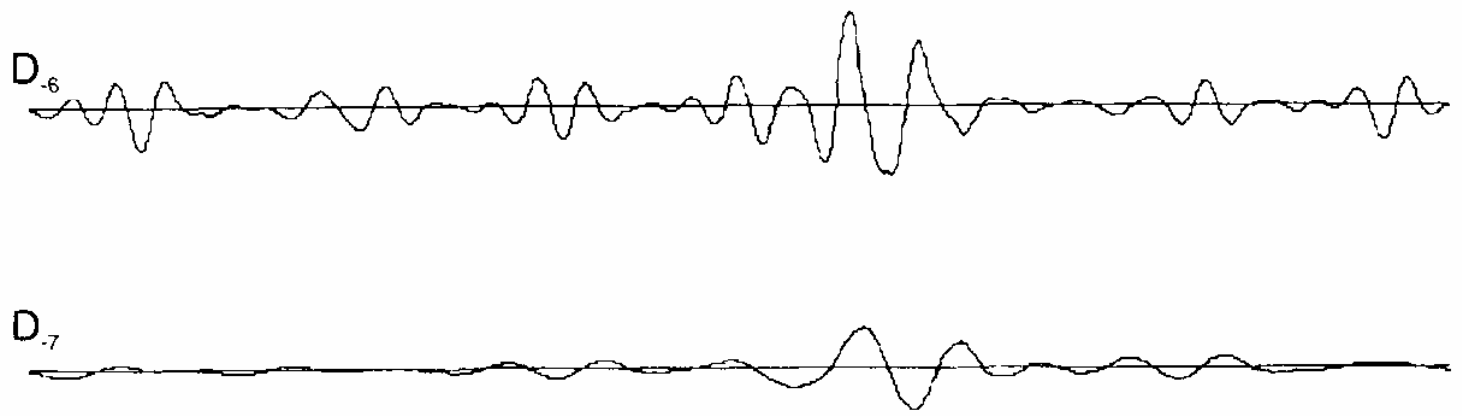

$\mathrm{D}_{-8}$

$\mathrm{D}_{-9}$

2. Orthonormal decomposition of an isolated beat. From the top (the original signal) to bottom (the details D-1 to D9). The two first levels contain the high frequency in- formation. The levels 3 and 4 hold the information related to the $\mathrm{P}$ and $\mathrm{QRS}$ waves. The $\mathrm{T}$ waves appear only on the lowest levels. 

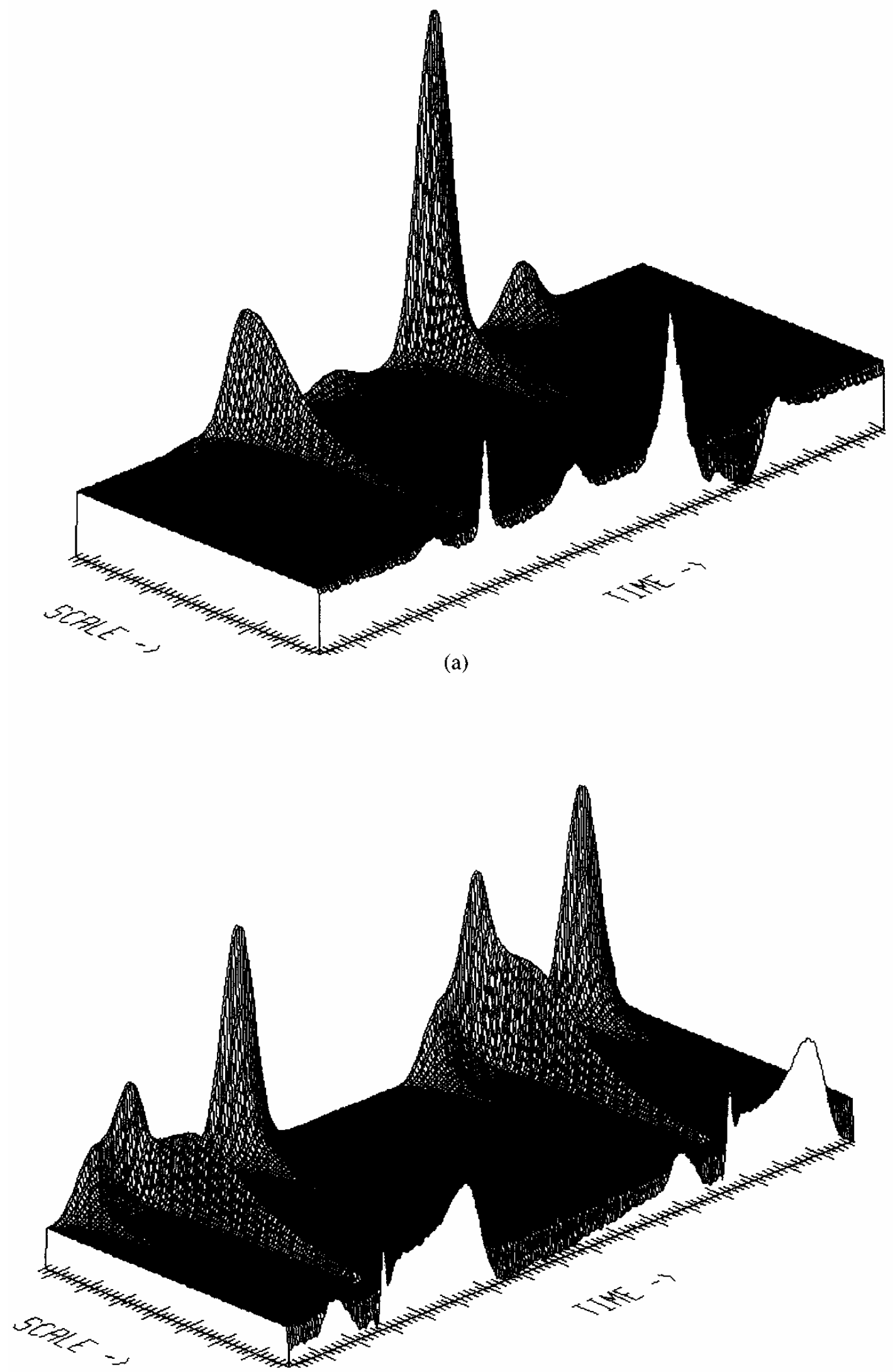

(b)

3. Time scale representation of cardiac beats by means of the complex wavelet (square modulus as a function of time and scale ; the original signal is on the time axis). (a) premature ventricular contraction; (b) two ischemic patterns depicting an elevation of the ST segment. 

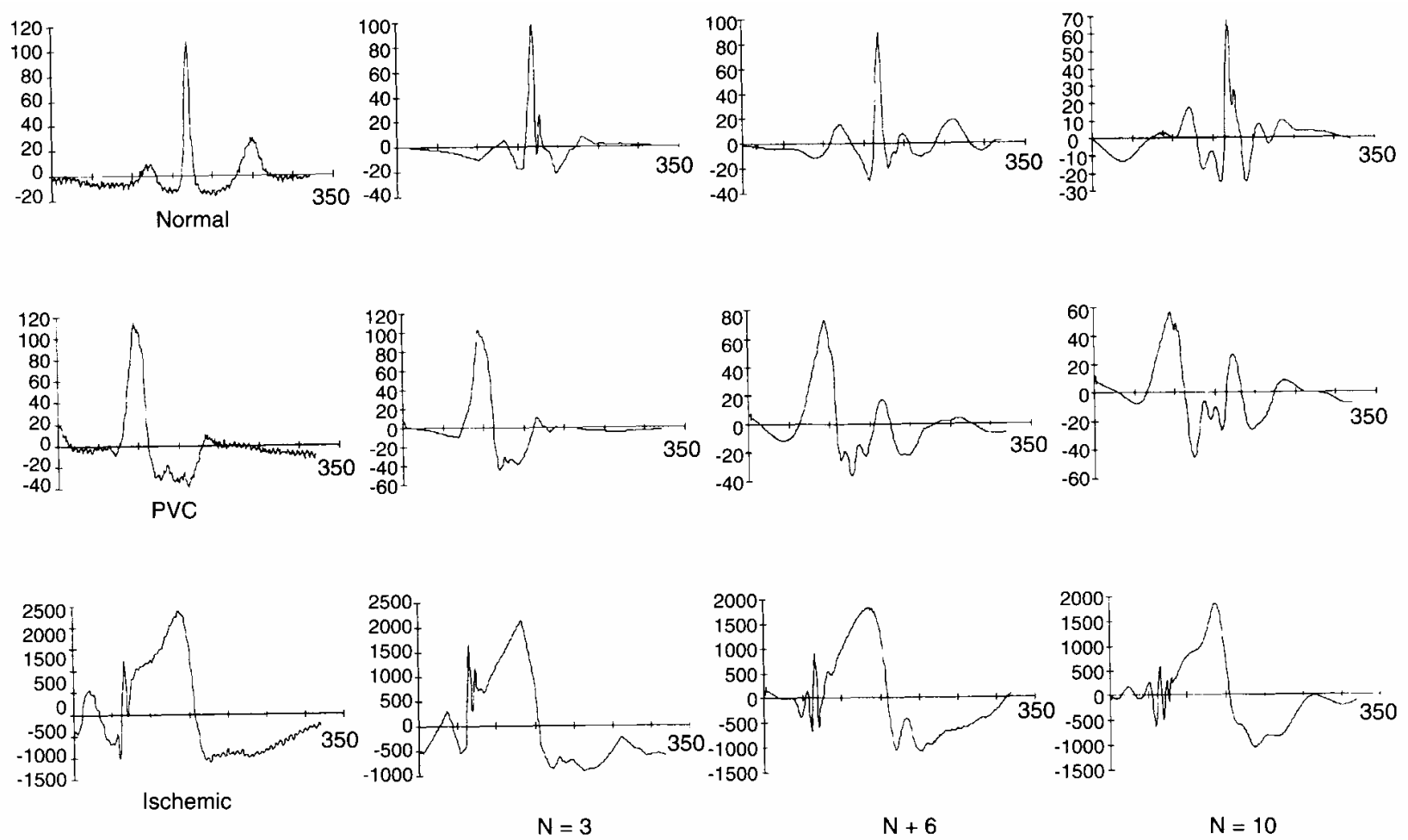

4. Reconstruction waveforms from the extrema (value and time occurrence) of the decomposition. The first column shows the three patterns (from top to bottom: normal, PVC, and ischemic beats). The remaining columns (left to right) define the reconstructed signals using different filter length $(\mathrm{N}=3,6$, and 10, respectively (see text)). 


\begin{tabular}{|c|c|c|c|}
\hline & \multicolumn{3}{|c|}{ Resulting Allocation } \\
\hline Initial Group & Normal (Nor) & $\begin{array}{c}\text { Premature ventricular } \\
\text { contraction (Pvc) }\end{array}$ & $\begin{array}{c}\text { Ischemic } \\
\text { beats (Isc) }\end{array}$ \\
\hline Normal & 20 & 0 & 0 \\
\hline $\begin{array}{c}\text { Premature ventricular } \\
\text { contraction }\end{array}$ & 1 & 12 & 0 \\
\hline Ischemic beats & 1 & 1 & 19 \\
\hline
\end{tabular}

Table 1: Classifying Waveforms (columns) with Respect to Original Groups (rows).

\begin{tabular}{|c|c|c|c|}
\hline & $\mathrm{N}=3$ & $\mathrm{~N}=6$ & $\mathrm{~N}=10$ \\
\hline Nor & 0.860 & 0.871 & 0.750 \\
\hline Pvc & 0.937 & 0.778 & 0.746 \\
\hline Isc & 0.952 & 0.958 & 0.916 \\
\hline
\end{tabular}

Table 2: Normalized Correlation Index between Original and Reconstructed Waveforms.

\begin{tabular}{|c|c|c|c|c|}
\hline & Raw Signal & Reconst. $\mathrm{N}=3$ & Reconst. N = 6 & Reconst. $\mathrm{N}=10$ \\
\hline Nor - Pvc & 0.608 & 0.685 & 0.328 & 0.289 \\
\hline Nor - Isc & 0.250 & 0.303 & 0.197 & 0.185 \\
\hline Pvc - Isc & 0.994 & 0.622 & 0.681 & 0.671 \\
\hline
\end{tabular}

Table 3: Maximal Correlation Index between Normal and Abnormal 1 Waveforms in Figure 4.

$\begin{array}{lcccc} & \text { Maximum } & \text { Mean } & \text { Minimum } & \text { Max - Min } \\ \text { Wavelet }(1) ; \mathrm{N}=6 & 100 & 95 & 89 & 11 \\ \text { Spline Wavelet } & 100 & 96 & 90 & 10 \\ \text { Morlet Type Wavelet } & 93 & 82 & 68 & 25 \\ \text { Temporal Features } & 96 & 83 & 64 & 32 \\ \text { Spectral Parameters } & 92 & 85 & 60 & 32\end{array}$

Table 4: Maximal Mean and Minimal Allocation Rates (Percent) from Various Wavelets and Descriptions. 